Document downloaded from:

http://hdl.handle.net/10251/177287

This paper must be cited as:

Riccio, J.; Alcaine, A.; Rocher-Ventura, S.; Laguna, P.; Saiz Rodríguez, FJ.; Martínez, JP. (2021). Omnipolar EGM Voltage Mapping for Atrial Fibrosis Identification Evaluated with an Electrophysiological Model. IEEE. 920-924.

https://doi.org/10.23919/Eusipco47968.2020.9287670

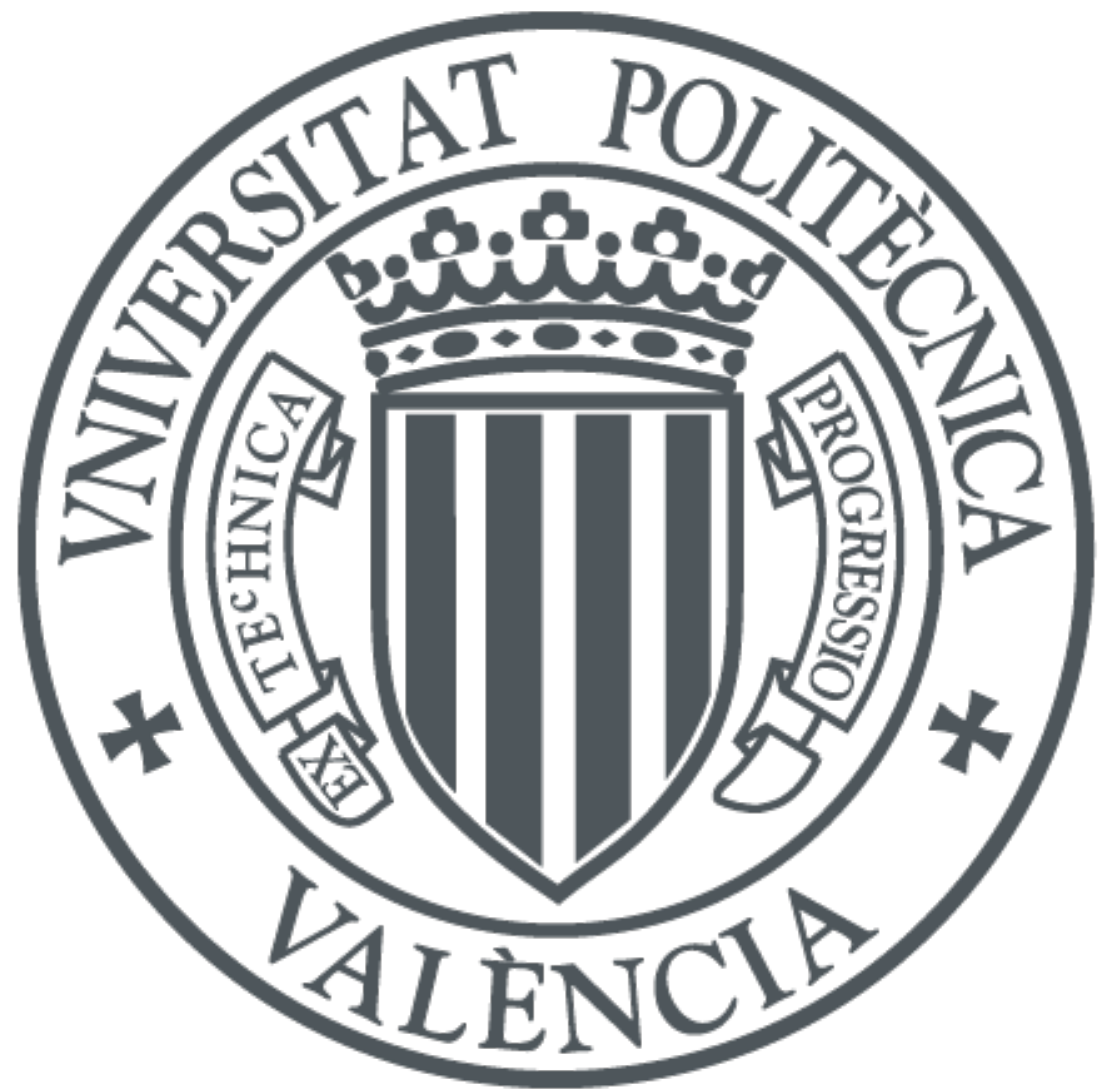

The final publication is available at

https://doi.org/10.23919/Eusipco47968.2020.9287670

Copyright IEEE

Additional Information 


\section{Omnipolar EGM Voltage Mapping for Atrial Fibrosis Identification Evaluated with an Electrophysiological Model}

\author{
Jenifer Riccio \\ I3A, IIS Aragón, CIBER-BBN \\ Universidad de Zaragoza \\ Zaragoza, Spain \\ jenriccio@unizar.es
}

\author{
Pablo Laguna \\ I3A, IIS Aragón, CIBER-BBN \\ Universidad de Zaragoza \\ Zaragoza, Spain \\ laguna@unizar.es
}

\author{
Alejandro Alcaine \\ I3A, IIS Aragón, CIBER-BBN \\ Universidad de Zaragoza \\ Zaragoza, Spain \\ aalcaineo@unizar.es
}

\author{
Javier Saiz \\ Centro de Inv. e Innov. en Bioingen. \\ Universitat Politècnica de València \\ Valencia, Spain \\ jsaiz@ci2b.upv.es
}

\author{
Sara Rocher \\ Centro de Inv. e Innov. en Bioingen. \\ Universitat Politècnica de València \\ Valencia, Spain \\ saroven94@gmail.com
}

\author{
Juan Pablo Martínez \\ I3A, IIS Aragón, CIBER-BBN \\ Universidad de Zaragoza \\ Zaragoza, Spain \\ jpmart@unizar.es
}

\begin{abstract}
Atrial fibrillation (AF) is the most spread heart arrhythmia, whose mechanisms are not completely clear yet. Catheter ablation is a standard treatment, which isolates the area involved in the arrhythmia. Intracardiac electrograms (EGMs) are used to better understand the AF mechanisms and to find appropriate ablation sites. Bipolar EGMs (b-EGMs) are often employed, but their amplitude and shape depend on catheter orientation, limiting reliability. To avoid this uncertainty, an approach insensitive to catheter orientation, referred as Omnipolar EGM (OP-EGM) method, has been introduced, which uses an estimation of the electric field within a group of electrodes, referred as clique. In this work, we compare different mapping approaches based on b-EGMs and OPEGM signals in simulation including fibrosis, so to evaluate their ability to detect fibrosis and reproduce the spatial distribution of the voltage. Maps have been computed using two clique configurations (square and triangular), introducing or not a previous time alignment of the bEGMs. OP-EGM signals have been obtained by projecting the electric field along directions of its maximal excursion and its principal components. Results show that both cliques configurations present good performance, in terms of fibrosis detection and correlation with the reference voltage maps. In addition, the proposed alignment of b-EGMS improves maps based on OP-EGM signals, especially when square cliques are used.
\end{abstract}

Index Terms-atrial fibrillation, fibrosis, electrograms, electroanatomical mapping, multi-electrode array, omnipolar, clique.

\section{INTRODUCTION}

Atrial Fibrillation (AF) is the most commonly diagnosed cardiac arrhythmia. It is characterized by an irregular electrical activation of the atria [1], whose frequency is higher than in normal sinus rhythm (SR). Catheter ablation of AF is the first line therapy when antiarrhythmic drug therapy is not effective, aimed at either eliminating the trigger or modifying the arrhythmogenic substrate. The most common ablation strategy consists of the electrical isolation of the pulmonary veins, often responsible of initiation and/or perpetuation of AF. Various degrees of atrial remodeling are associated with AF. Structural remodeling generates atrial fibrosis, which represents an anchor site for rotors and facilitates their formation, creating a substrate for AF [2].

Electrophysiologically, atrial fibrosis produces lowamplitude bipolar electrograms (b-EGMs), EGM fractionation and slower conduction velocity [3]. These abnormal manifestations can be identified using electroanatomical mapping (EAM). Most ablation procedures targeting low-voltage areas perform EAM using a threshold of $0.5 \mathrm{mV}$ (peak-to-peak voltage of b-EGM) during SR. However, b-EGMs amplitude depends on the relative orientation between the travelling voltage wavefront and the recording electrodes pair, possibly leading to misguided ablation strategies [4]. In order to overcome this limitation, an orientation-independent 
approach, referred as Omnipolar EGM (OP-EGM), has been proposed [5]. This technique estimates the local electric field from the EGMs recorded at groups of nearby electrodes to find propagation and substrate parameters.

In this work, we study and compare different strategies to obtain voltage maps based on the OP-EGM approach in the context of a simulation study. We assess the ability of each mapping strategy to reproduce the unipolar voltage maps, as well as their ability to identify a fibrosis patch. Two different catheter orientations with respect to the electrical propagation have been studied, so as to assess their sensitivity to catheter orientation.

\section{MATERIALS}

A 2-D atrial tissue of $4 \times 4 \mathrm{~cm}$ of hexahedric elements has been simulated with $100 \mu \mathrm{m}$ resolution using the Courtemanche cellular model [6] with conduction heterogeneity of left atrium and remodeling induced by chronic AF. A circular patch of diffuse fibrosis having a diameter of $2 \mathrm{~cm}$ and a random distribution of $20 \%$ has been defined within the tissue. Nodes have been assigned the Maleckar fibroblast model and a conduction reduction of $30 \%$.

Unipolar EGMs (u-EGMs) have been computed $v_{i, j}^{u}(t)$ with a sampling frequency of $1 \mathrm{kHz}$ at the sites $(i, j) \quad(i, j \in\{1, . ., 15\})$ corresponding to a high-density multi-electrode array (MEA) of $15 \times 15$ electrodes with inter-electrode distance $d=2 \mathrm{~mm}$, centered in the tissue slice, at $1 \mathrm{~mm}$ distance from the tissue surface. Each simulated u-EGM is $500 \mathrm{~ms}$ long and contains a single activation (corresponding to one sinus beat). Bipolar b-EGMs are derived form the unipolar as $v_{i, j}^{h}(t)=v_{i, j}^{u}(t)-v_{i+1, j}^{u}(t)$, for the horizontal and $v_{i, j}^{v}(t)=v_{i, j}^{u}(t)-v_{i, j+1}^{u}(t)$ for the vertical.

\section{METHODS}

\section{A. OP-EGM approach and signals}

In this work, a peak-to-peak voltage estimation has been performed using the OP-EGM approach, which relates the spatial gradient of the unipolar voltage $\phi(t)$ at each electrode, $v_{i, j}^{u}(t)$, with the electric field $\mathbf{E}(t)$ at the extracellular-myocardial interface: $\mathbf{E}(t)=-\nabla \phi(t)$ [5].

The OP-EGM method assumes a locally plane and homogeneous wave travelling within each group of three or four nearby electrodes of the MEA, referred as clique. For each clique, $\mathbf{E}(t)$ is estimated from the different b-EGMs in the clique using least squares estimates. The local electric field evolution over the time of the activation front describes a loop trajectory in the plane defined by each clique. The assumption of locally plane and homogeneous wave implies that $\mathbf{E}(t)$ has a constant direction and the loop lies in a straight line. If the wave is not plane $\mathbf{E}(t)$ lies in a bidirectional plane, suggesting that the projection of $\mathbf{E}(t)$ into a single direction is not enough to characterize the propagation.

Two possible 2-D clique configurations were considered in this work: square and triangular, with four and three electrodes, respectively. The square configuration provides one single measurement point for each group of four electrodes at positions $\quad[(i, j),(i, j+1),(i+1, j),(i+1, j+1)]$, $i=1, \ldots, 14 ; j=1, \ldots, 14$, giving a total of $14 \times 14=196$ OP-EGM signals, $v_{i, j}^{\text {op }}(t)$, within the considered MEA. On the other hand, the triangular configuration provides 4 measurement points for each group of four electrodes, resulting in a total of $4 \times 196=784$ OP-EGM signals, $v_{i, j, q}^{\mathrm{OP}}(t)$, being $q \in\{1,2,3,4\}$ the quadrant from bottom left, to bottom right, to up left and to up right.

It can be easily shown that, both in square and triangular configurations, the least square estimate of $\mathbf{E}_{i, j}(t)$ is a linear combination of horizontal, $v_{i, j}^{h}(t)$, and vertical, $v_{i, j}^{v}(t)$, b-EGMs obtained within the clique electrodes. For the square clique configuration, $\mathbf{E}_{i, j}(t)=\left[\left(v_{i, j}^{h}(t)+\right.\right.$ $\left.\left.v_{i, j+1}^{h}(t)\right) \mathbf{u}_{h}+\left(v_{i, j}^{v}(t)+v_{i+1, j}^{v}(t)\right) \mathbf{u}_{v}\right] / 2 d$, where $\mathbf{u}_{h}$ and $\mathbf{u}_{v}$ are unitary vectors in horizontal and vertical directions. For triangular clique configuration, $\mathbf{E}_{i, j, 1}(t)=$ $\left[v_{i, j}^{h}(t) \mathbf{u}_{h}+v_{i, j}^{v}(t) \mathbf{u}_{v}\right] / d, \mathbf{E}_{i, j, 2}(t)=\left[v_{i, j}^{h}(t) \mathbf{u}_{h}+\right.$ $\left.v_{i+1, j}^{v}(t) \mathbf{u}_{v}\right] / d, \mathbf{E}_{i, j, 3}(t)=\left[v_{i, j+1}^{h}(t) \mathbf{u}_{h}+v_{i, j}^{v}(t) \mathbf{u}_{v}\right] / d$ and $\mathbf{E}_{i, j, 4}(t)=\left[v_{i, j+1}^{h}(t) \mathbf{u}_{h}+v_{i+1, j}^{v}(t) \mathbf{u}_{v}\right] / d$.

$\mathbf{E}(t)$ is therefore estimated from b-EGM obtained from different pairs of electrodes which are activated at different times. When the direction of propagation is not parallel to one of the MEA axes, deflections at those b-EGMs are disaligned, resulting in a disalignment between the estimated horizontal and vertical components of $\mathbf{E}(t)$ which widens the loop and affects the electric field direction. To avoid this effect, a modified version of the least square estimator of $\mathbf{E}(t)$ has been implemented by performing previous time alignment of b-EGMs. Examples of electric fields estimated in square and triangular cliques, together with their respective bEGMs and OP-EGM signals, are shown in Fig. 1.

We also used two different approaches to obtain OP-EGM signals as projections of $\mathbf{E}(t)$ within one clique: a) $v_{i, j}^{\text {OP-ME }}(t)=d \mathbf{E}_{i, j}(t) \cdot \mathbf{u}_{m}$, where $\mathbf{u}_{m}$ is is the unitary vector in the direction of maximal excursion (ME) within an activation, as described in [5] and b) $v_{i, j}^{\text {OP-PCA }}(t)=d \mathbf{E}_{i, j}(t) \cdot \mathbf{u}_{\nu}$, where $\mathbf{u}_{\nu}$ is the unitary vector in the principal direction obtained by Principal Compo- 

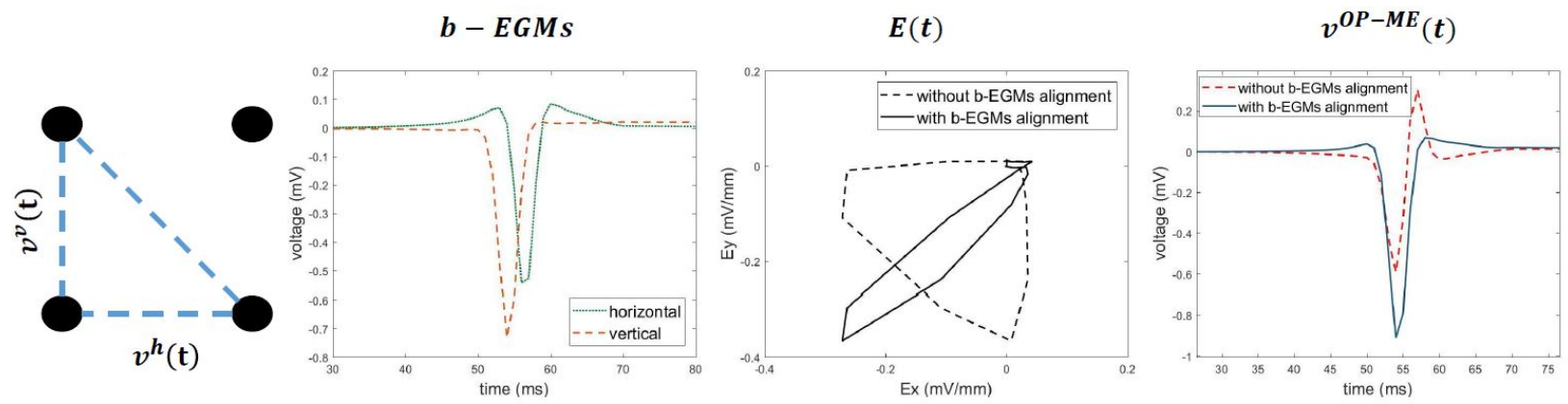

Fig. 1: From left to right: example of triangular clique with respective b-EGMs $v^{h}(t)$ and $v^{v}(t)$, loops of $\mathbf{E}(t)$ (obtained with/without previous alignment of b-EGMs) and OP-EGM $v^{\text {OP-ME }}(t)$ got as projection of the electric field along the direction of its maximal excursion. $(i, j)=(4,13)$ from propagation parallel to vertical direction.

nent Analysis (PCA) of the loop described by $\mathbf{E}_{i, j}(t)$. In this approach, we also obtained the voltage projected in the orthogonal direction $\mathbf{u}_{\nu}^{\perp}$ (corresponding to the second principal component): $v_{i, j}^{\text {op-PCA } \perp}(t)=d \mathbf{E}_{i, j}(t) \cdot \mathbf{u}_{\nu}^{\perp}$.

\section{B. Voltage maps}

For each OP-EGM signal $v_{i, j}^{\text {OP-X }}(t)$, $\mathrm{X} \in\{\mathrm{ME}, \mathrm{PCA}, \mathrm{PCA} \perp\}$, we compute the peak-to-peak voltage, $\quad V_{i, j}^{\text {op-X }}=\max _{t}\left\{v_{i, j}^{\text {op-X }}(t)\right\}-\min _{t}\left\{v_{i, j}^{\text {op-X }}(t)\right\}$, that we use for voltage mapping. In the PCA approach, we created maps based on the root sum square (RSS) of the voltage in the first principal component and the orthogonal direction

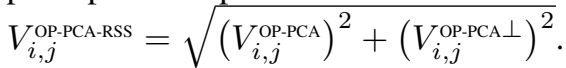

Voltage maps were computed for each orientation of the MEA $\left(0^{\circ}\right.$ or $\left.45^{\circ}\right)$ and clique configuration (square or triangular). For each MEA orientation, we also built voltage maps based on the peak-to-peak voltage of the bEGMs in each of the two catheter directions (i.e. horizontal and vertical), $V_{i, j}^{x}=\max _{t}\left\{v_{i, j}^{x}(t)\right\}-\min _{t}\left\{v_{i, j}^{x}(t)\right\}$, $x \in\{h, v\}$. Additionally, the RSS of both values, $V_{i, j}^{b-\text { rss }}=\sqrt{\left(V_{i, j}^{h}\right)^{2}+\left(V_{i, j}^{v}\right)^{2}}$, as well as their maximum $V_{i, j}^{b-\max }=\max \left\{V_{i, j}^{h}, V_{i, j}^{v}\right\}$ are considered.

\section{Assessment of correlation with unipolar voltage map}

In order to evaluate and compare the voltage maps obtained with different OP-EGM approaches, we built a reference voltage map based on the peak-to-peak voltage of the u-EGM, $V_{i, j}^{u}=\max _{t}\left\{v_{i, j}^{u}(t)\right\}-\min _{t}\left\{v_{i, j}^{u}(t)\right\}$. The reference voltage map was resampled with a bi-cubic interpolation to match the positions of the voltage values obtained in bipolar and omnipolar maps.
To quantify the agreement of each bipolar and omnipolar voltage map with the substrate characterization given by the reference voltage map, we used both Pearson $\rho_{p}$ and Spearman's rank $\rho_{s}$.

\section{Assessment of voltage maps for fibrosis detection}

Receiver operating characteristic (ROC) curves have been used in order to evaluate the ability of the proposed voltage maps in identifying the fibrotic tissue area, discriminating it from the healthy area. For that purpose, a ground-truth binary mask of fibrosis has been created setting to 1 all the cliques whose centre was within the simulated fibrosis patch and to 0 all the cliques whose centre was in the healthy area.

For each proposed voltage map, thresholds for fibrosis identification have been swept in order to compute the ROC curve, obtaining the sensitivity (Se) and specificity $(\mathrm{Sp})$ in the detection of the fibrotic areas. The area under the curve (AUC) has been estimated for each map as a measurement of its overall fibrosis detection ability. In addition, the values of Se and Sp have been computed for the threshold with maximum accuracy (ACC), defined as the number of correctly identified cliques (fibrosis or healthy tissue) divided by the total number of cliques.

\section{Results}

Table I shows the overall results of fibrosis detection and correlation with unipolar voltage maps in the simulated tissue, considering both vertical $\left(0^{\circ}\right)$ and oblique $\left(45^{\circ}\right)$ MEA directions.

Bipolar maps considering both horizontal and vertical b-EGMs (either by keeping the maximum value or computing the RSS value) are able to identify the fibrotic area with ACC of $88 \%$ and $87 \%$ respectively. 


\section{$\Lambda$}

Propagation direction

a)

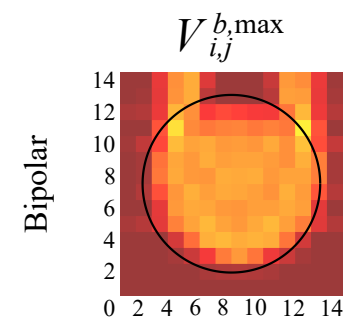

I

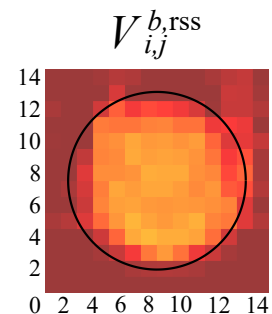

Propagation direction

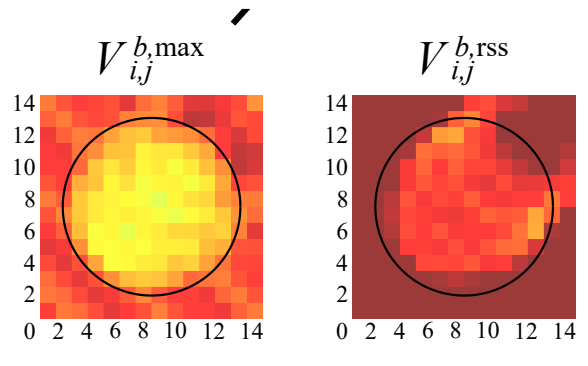

b)

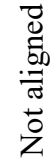

Square Cliques

$V_{i, j}^{\mathrm{OP}-\mathrm{ME}}$

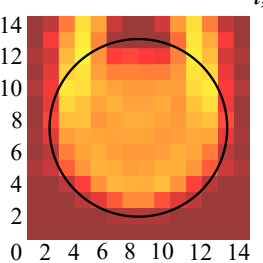

$\begin{array}{llllllll}0 & 2 & 4 & 6 & 8 & 10 & 12 & 14\end{array}$

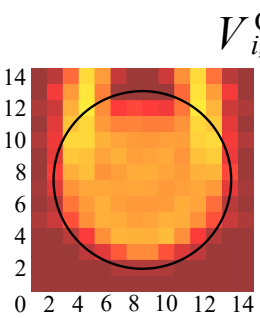

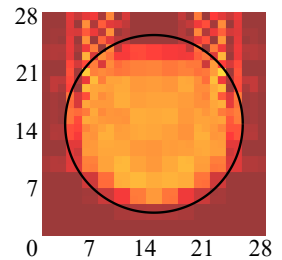

0
$-P C A$

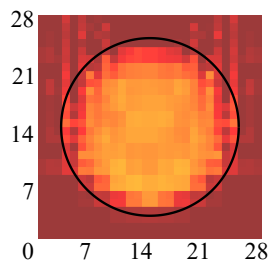

Square Cliques

Triangular Cliques

$V_{i, j}^{\mathrm{OP}-\mathrm{ME}}$
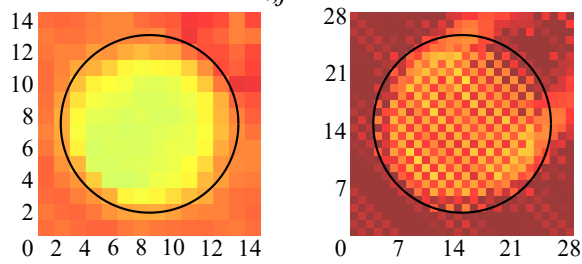

$$
V_{i, j}^{\mathrm{OP}-\mathrm{PCA}}
$$
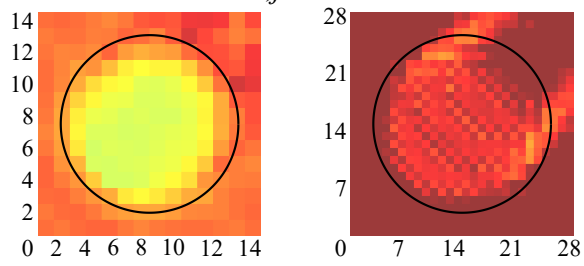

$V_{i, j}^{\mathrm{OP}-\mathrm{ME}}$

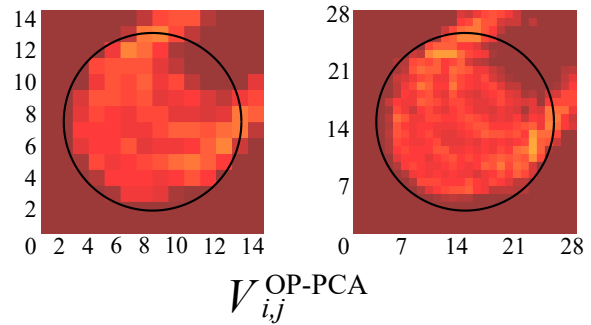

c)

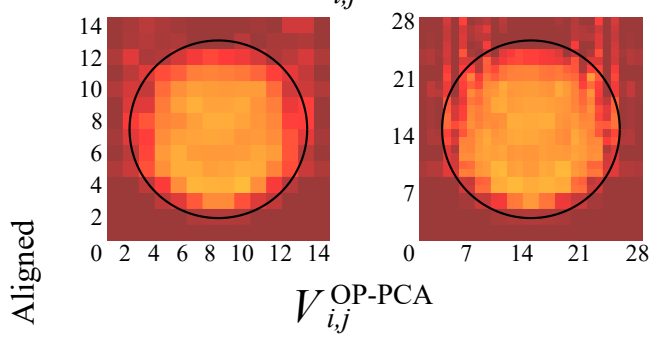

$\mathrm{t}$
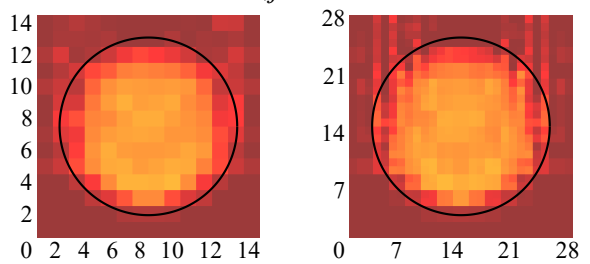
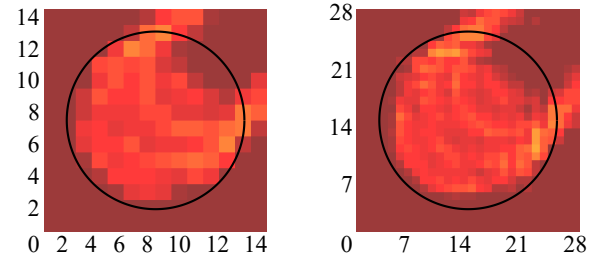

Fig. 2: Results when MEA is $0^{\circ}$ (left column) and $45^{\circ}$ degrees (right column) oriented. In both columns: (a): Maps of the maximum, $V_{i, j}^{b, \max }$, (left) and of $V_{i, j}^{b, \text { rss }}$, (right); (b): Voltage maps from of OP-EGM signals: $V_{i, j}^{\text {OP-ME }}$ (top) and $V_{i, j}^{\text {op.PA }}$ (bottom) by using square (left) and triangular (right) cliques configurations; (c): Voltage maps obtained from OP-EGM signals as (b) but after aligning b-EGMs. In each map, fibrosis patch has been pictured as a black circumference. All the voltage values have been mapped in range between $0 \mathrm{mV}$ and $1 \mathrm{mV}$. 
TABLE I: Detection performance of peak-to-peak voltage maps including vertical and oblique MEA orientation

\begin{tabular}{|c|c|c|c|c|c|c|c|c|c|c|}
\hline Electrograms & Map type & cliques & alignment & Threshold (mV) & $\mathrm{Se}(\%)$ & $S p(\%)$ & $A C C(\%)$ & $A U C$ & $\rho_{p}^{*}$ & $\rho_{s}^{*}$ \\
\hline \multirow{2}{*}{ b-EGMs } & $V_{i, j}^{b-\max }$ & - & - & 0.78 & 82 & 93 & 88 & 0.91 & 0.75 & 0.76 \\
\hline & $V_{i, j}^{b-\text { rss }}$ & - & - & 0.93 & 81 & 93 & 87 & 0.93 & 0.82 & 0.82 \\
\hline \multirow{8}{*}{ OP-EGMs } & $V_{i, j}^{\mathrm{OP}-\mathrm{ME}}$ & square & no & 0.77 & 83 & 87 & 85 & 0.88 & 0.68 & 0.71 \\
\hline & $V_{i, j, q}^{\mathrm{OP}-\mathrm{ME}}$ & triangular & no & 0.90 & 85 & 89 & 87 & 0.92 & 0.80 & 0.81 \\
\hline & $V_{i, j}^{\text {OP-PCA-RSS }}$ & square & no & 0.77 & 81 & 88 & 85 & 0.88 & 0.68 & 0.71 \\
\hline & $V_{i, j, q}^{\text {OP-PCA-RSS }}$ & triangular & no & 0.97 & 83 & 87 & 85 & 0.91 & 0.77 & 0.78 \\
\hline & $V_{i, j}^{\mathrm{OP}-\mathrm{ME}}$ & square & yes & 0.93 & 87 & 93 & 90 & 0.94 & 0.82 & 0.83 \\
\hline & $V_{i, j, q}^{\mathrm{OP}-\mathrm{ME}}$ & triangular & yes & 0.97 & 88 & 86 & 87 & 0.93 & 0.81 & 0.81 \\
\hline & $V_{i, j}^{\text {OP-PCA-RSS }}$ & square & yes & 0.92 & 85 & 94 & 90 & 0.93 & 0.81 & 0.83 \\
\hline & $V_{i, j, q}^{\text {OP-PCA-RSS }}$ & triangular & yes & 0.97 & 86 & 87 & 87 & 0.92 & 0.80 & 0.81 \\
\hline
\end{tabular}

However, bipolar maps using RSS of both directions present a better correlation with the reference voltage $\operatorname{map}\left(\rho_{s}=0.82\right.$ for RSS vs $\rho_{s}=0.75$ for maximum).

Table I also shows that omnipolar maps obtained without previous alignment of the b-EGMs do not attain the performance obtained by the RSS bipolar map. However, when alignment is performed before obtaining the OP-EGMs, these maps achieve indeed comparable or better performance than the RSS of the b-EGMs. Within the different omnipolar approaches, it is observed that those based on square cliques are the ones that benefit the most from previous b-EGM alignment. After b-EGM alignment all the OP-based strategies present similar performance.

Figure 2 shows the voltage maps obtained by bipolar and omnipolar EGMS in the simulated tissue considered in this work for vertical and oblique propagations. One can there observe that the behavior of the different maps has important differences depending on the relative direction between the MEA and wave propagation.

\section{Discussion AND CONCLUSIONS}

In this work, an electrophysiological model has been used to simulate an atrial tissue with fibrosis. Different OP-EGM-based approaches have been compared in their ability to characterize the simulated atrial substrate. More in detail, performance of peak-to-peak voltage maps in detecting fibrosis and reproducing reference unipolar voltages has been evaluated, as well as their sensibility with the orientation of mapping catheter. All the proposed omnipolar maps show good performance in discriminating fibrotic tissue. When the MEA is parallel to the propagation direction, peak-to-peak voltage maps obtained from square cliques give the best outcomes, outperforming triangular cliques. The fact that a plane wavefront is present in the simulated tissue may be the reason for this result.

Results in this simulation study have shown that time alignment of the b-EGM proposed in this work improves the omnipolar voltage estimation. However, the use of PCA to determine the main directions in the electric field loop has not shown superiority with respect to the direction of maximal excursion. These results need to be complemented with other simulation configurations, as nonplanar or multiple wavefronts.

\section{ACKNOWLEDGMENT}

This work is part of a project that has received funding from the European Union's Horizon 2020 research and innovation program under the Marie Skłodowska-Curie grant agreement No 766082 (MY-ATRIA).

\section{REFERENCES}

[1] H. Calkins et al., 2017 HRS/EHRA/ECAS/APHRS/SOLAECE expert consensus statement on catheter and surgical ablation of atrial fibrillation. Heart Rhythm, vol.14(10), pp.e275-e444, 2017.

[2] B. Burstein and S. Nattel, "Atrial fibrosis: mechanisms and clinical relevance in atrial fibrillation," JACC, vol. 51(8), pp. 802809, 2008.

[3] M. Rodríguez-Mañero et al., "Validating left atrial low voltage areas during atrial fibrillation and atrial flutter Using multielectrode automated electroanatomic mapping," JACC: Clinical Electrophysiology, vol. 4(12), pp.1541-1552, 2018.

[4] S.K. Haldar et al., "Resolving bipolar electrogram voltages during atrial fibrillation using omnipolar mapping," Circ. Arrhythm. Electrophysiol., vol. 10(9), pp. 1-13, 2017.

[5] D.C. Deno et al., "Orientation-independent catheter-based characterization of myocardial activation," IEEE Trans. Biomed. Eng., vol. 64(5), pp. 1067-1077, 2017.

[6] M. Courtemanche, R.J. Ranirez and S. Nattel, "Ionic mechanisms underlying human atrial action potential properties: insights from a mathematical model," Am. J. Physiol., vol. 275(1), pp. H30121, 1998.

[7] J. Riccio et al., "Characterization of propagation patterns with omnipolar EGM in epicardial multi-electrode arrays," Proc. Computing in Cardiology, Singapore, vol. 46, pp. 1-4 2019. 